\title{
Atração do predador Doru luteipes (Scudder) (Dermaptera: Forficulidae) por estímulos olfativos de dietas alternativas em laboratório
}

\author{
Attraction of the predator Doru luteipes (Scudder) (Dermaptera: Forficulidae) by olfactory stimulus of \\ alternative diets in the laboratory
}

Mariana Campana Nonino ${ }^{\mathrm{I}}$ Amarildo Pasini ${ }^{\mathrm{II}}$ Maurício Ursi Ventura ${ }^{\mathrm{III}}$

\section{RESUMO}

Doru luteipes consome lagartas de Spodoptera frugiperda, que é atualmente a principal praga da cultura do milho no Brasil. Normalmente, se $70 \%$ das plantas tivessem o predador, a praga seria mantida abaixo do nível de dano econômico. Entretanto, o predador ocorre após a praga danificar as plantas. Assim, a disponibilidade de materiais que atraíssem e antecipassem a presença do predador na lavoura poderia reduzir populações da praga. Desta forma, estudou-se em laboratório a atratividade de dietas artificiais para machos e fêmeas do predador $\mathbf{D}$. luteipes. Os tratamentos foram: pupa desidratada e moída do bicho-da-seda; pólen misto, nas quantidades de 0,05; 0,1;0,2;0,5 e 1,0g para ambas as dietas. Em olfatômetro, os estímulos da dieta foram comparados à testemunha. Foram realizadas 30 repetições. Os resultados mostraram que houve atratividade tanto para machos quanto para fêmeas. Em geral, a atração foi aumentada com o aumento da quantidade das dietas. Estudos adicionais foram propostos para testar a viabilidade do uso dos materiais como estratégia de manejo da praga no campo.

Palavras-chave: Spodoptera frugiperda, Zea Mays, controle biológico e dermaptera.

\section{ABSTRACT}

Doru luteipes consumes larvae of Spodoptera frugiperda, which is at this moment the main pest of maize crop in Brazil. In general, if $70 \%$ of the plants had this predator the pest would be kept under the threshold of economic damage. However, the predator occurs after the pest damages the plants. Thus, the availability of materials that would attract and anticipate the presence of predator in the field,could reduce pest populations. In such a way, it was studied in the laboratory the attractiveness of artificial diets for males and females of the D. luteipes. The treatments were: ground dehydrated pupa of the silk worm and pollen mixture in the amounts of 0.05, 0.1, $0.2,0.5$ e $1.0 \mathrm{~g}$, for both the diets The diet stimulus were compared to control using a olfactometer. In general, attraction increased with rising amounts of the diets. Further investigations were proposed to test the viability of the usage of the materials to manage the pest in the field.

Key words: Spodoptera frugiperda, Zea Mays, biological control and dermaptera.

\section{INTRODUÇÃO}

Com a expansão da cultura do milho (Zea mays L.) no Brasil, os problemas com pragas também têm aumentado. A lagarta-do-cartucho, Spodoptera frugiperda (J. E. Smith), é relatada como a principal praga de importância econômica, podendo causar danos de até 35\% (CRUZ et al., 1996). O uso indiscriminado destes produtos causa o desequilíbrio biológico, eliminando os inimigos naturais e elevando a população da praga (CRUZ, 1995).

O controle biológico possui grande potencial de utilização em lavouras de milho. Para sua utilização prática, inovações nas técnicas de produção, utilização de nutrientes artificiais, melhoramento no

IPrograma de Pós-graduação em Agronomia, Londrina, PR, Brasil.

IIDepartamento de Agronomia, Centro de Ciências Agrárias, Universidade Estadual de Londrina (UEL), Londrina, PR, Brasil.

IIIDepartamento de Agronomia, Centro de Ciências Agrárias, UEL, CP 6001, 86051-970, Londrina, PR, Brasil. E-mail: mventura@uel.br. Autor para correspondência. 
habitat, liberações de inimigos naturais em época correta, manipulação daqueles já existentes, técnicas de preservação e uso de espécies ou raças mais efetivas contribuem para incrementar sua eficiência (PARRA et al., 2002).

As tesourinhas, dentre as quais se inclui a espécie Doru luteipes (Scudder), têm sido citadas por estarem entre os organismos mais eficazes no controle de $\boldsymbol{S}$. frugiperda pelo grande consumo da presa, estimado em 21 e 12 ovos por dia para adultos e ninfas, respectivamente (CRUZ, 1995). Este predador de hábito noturno foi citado por ALVARENGA et al. (1996) por possibilitar a integração do controle biológico com a resistência de plantas de sorgo (Sorghum bicolor L). Em algodoeiro (Gossypium hirsutum L), foi citado por CAMPOS \& GRAVENA (1984) no controle de Heliothis spp.

LEITE \& LARA (1985) verificaram a flutuação populacional de pragas e inimigos naturais associados à cultura da soja e observaram que o predador ( $\boldsymbol{D}$. lineare) encontrava-se em abundância principalmente no início da fase de florescimento.

A presença do predador em $70 \%$ das plantas de milho seria o suficiente para manter a lagarta-docartucho sob controle, ficando abaixo do nível de dano econômico (WAQUIL et al., 2002). Entretanto, sua ocorrência no campo é tardia, ou seja, comumente após o pico populacional da praga (PASINI, 2002). Os agentes naturais de controle normalmente apresentam este padrão de resposta populacional dependente da densidade do hospedeiro ou da presa. Seria interessante que se pudesse dispor de materiais que atraíssem e mantivessem os agentes de controle biológico antes do pico populacional da praga no campo, para que pudessem, desta forma, reduzir efetivamente suas populações.

Assim, o objetivo do trabalho foi avaliar em laboratório a atratividade de dietas artificiais para machos e fêmeas do predador Doru luteipes.

\section{MATERIAL E MÉTODOS}

O experimento foi realizado no laboratório de Entomologia da Universidade Estadual de Londrina (UEL), em Londrina, Paraná. As coletas de D. luteipes visando à criação estoque iniciaram-se em fevereiro de 2005, em diferentes áreas de milho, sendo posteriormente mantidas a $25 \pm 2^{\circ} \mathrm{C}$; $60 \% \mathrm{UR} ; 14 \mathrm{~h}$ fotofase. A metodologia de criação foi baseada em
PASINI et al. (2003a). As tesourinhas foram colocadas em placa de acrílico e utilizou-se canudinho transparente de refrigerante com algodão umedecido para oviposição e abrigo. A dieta artificial para manutenção foi à base de pupa desidratada e moída do bicho-da-seda (Bombyx mori) e de pólen misto de plantas coletado por Apis mellifera. O alimento foi ofertado em forminhas laminadas. A cada três dias, o algodão era umedecido e a alimentação trocada (PASINI et al., 2003a).

Para os bioensaios, utilizou-se um olfatômetro em forma de "Y”. Neste aparato, os insetos caminhavam contra o fluxo de ar gerado por bomba de aquário, e podiam optar entre um braço com estímulo e outro sem (testemunha). Antes do início do experimento, os adultos foram mantidos em jejum por 72 horas, apenas com água destilada. Os tratamentos foram: pupa desidratada e moída do bicho-da-seda ( $\boldsymbol{B}$. mori); pólen misto de plantas (coletado por $\boldsymbol{A}$. mellifera), nas seguintes quantidades: 0,$05 ; 0,1 ; 0,2$; 0,5 e $1,0 \mathrm{~g}$, para ambas as dietas.

Foram realizadas 30 repetições para fêmeas e para machos. Os resultados foram analisados estatisticamente pelo Teste dos Sinais e Meta -Análise para Várias Proporções (AYRES et al., 2003).

\section{RESULTADOS E DISCUSSÕES}

Fêmeas de $\boldsymbol{D}$. luteipes foram atraídas por farinha de pupa moída de $\boldsymbol{B}$. mori $(1,0$ e $0,2 \mathrm{~g})$ em olfatômetro (Figura 1). Maiores quantidades de farinha $(1,0 \mathrm{~g})$ proporcionaram capturas significativamente maiores do que a menores quantidades $(0,05 \mathrm{~g})$ (Tabela 1). Para machos, somente na quantidade de $1 \mathrm{~g}$ de farinha, foi observada atração (Figura 2). Com esta quantidade, também foram observadas diferenças significativas em relação à menor quantidade $(0,05 \mathrm{~g})$ (Tabela 1).

Pólen misto, coletado por A. mellifera, atraiu fêmeas de $\boldsymbol{D}$. luteipes quando quantidades de 1,0 e 0,2g foram utilizadas no olfatômetro (Figura 3). Quantidades de 1,0; 0,5 e 0,2g atraíram os machos em olfatômetro (Figura 4). Tanto para machos quanto para fêmeas, constatou-se maior número de adultos atraídos para as quantidades maiores de farinha (1,0; 0,5 e 0,2g) do que para as menores $(0,05 \mathrm{~g})$ (Tabela 1$)$. Normalmente, o pólen tem sido citado como uma substância doce e os açúcares são fagoestimulantes para a maioria dos insetos (DITMAN \& CORY, 1933; ADLER, 1989; BARTLET et al.. , 1994). 


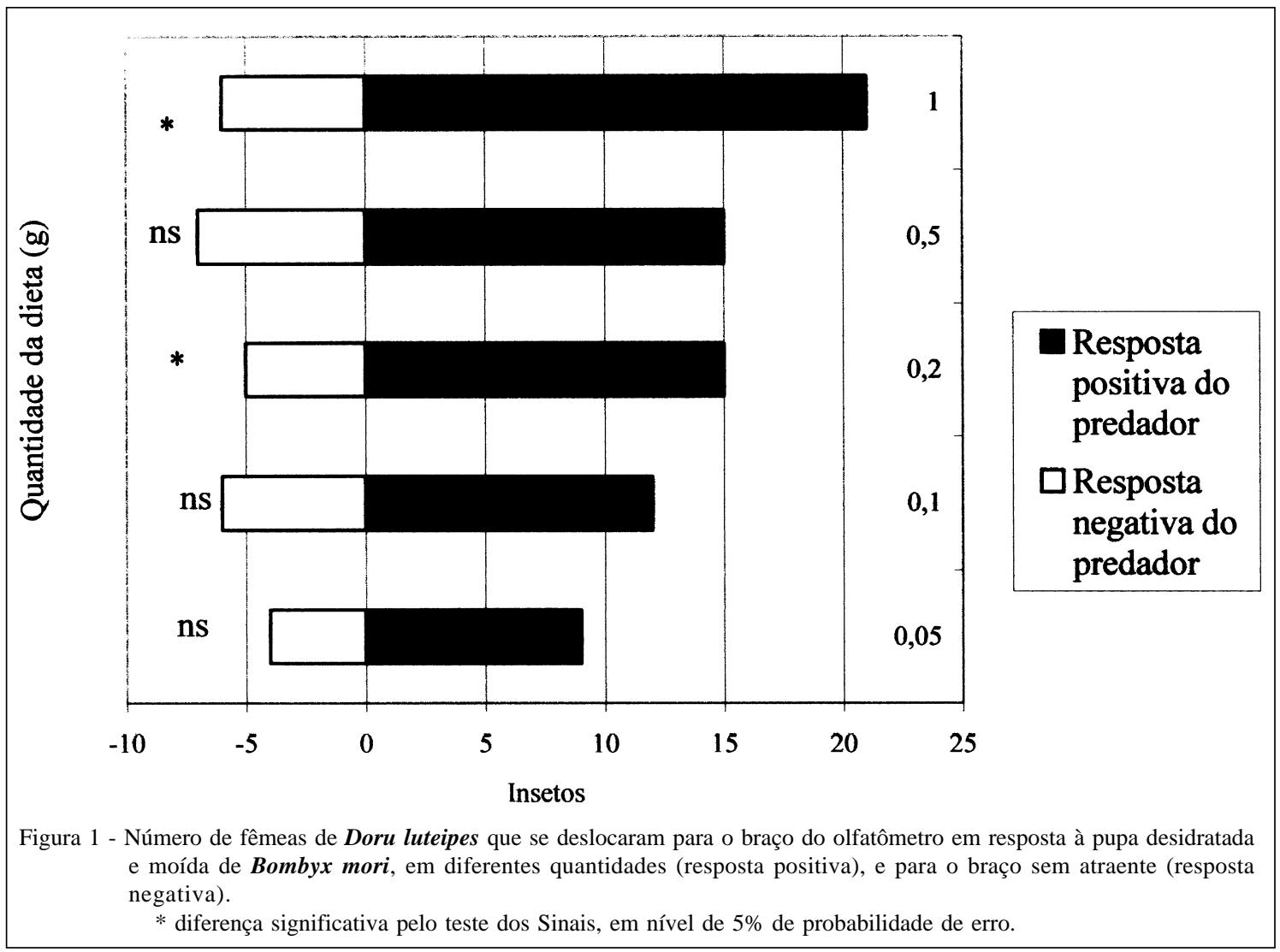

Portanto, verificou-se que machos e fêmeas de $\boldsymbol{D}$. luteipes foram atraídos pelos alimentos, havendo uma tendência de incremento das capturas à medida que se aumentavam as doses (Figuras 1 a 4, Tabela 1). Em estudo anterior, a população de Doru sp., criada com pupa desidratada e moída de $\boldsymbol{B}$. mori misturada a pólen de taboa (Typha angustiflolia), apresentou 100\% de viabilidade ninfal (PASINI ET AL., 2003b). Sendo assim, verifica-se que os materiais utilizados são adequados para avaliações no campo com o propósito de incrementar as populações da tesourinha.

Tabela 1 - Número de machos e fêmeas de Doru luteipes que se deslocaram para o braço do olfatômetro em resposta a estímulos olfativos de pólen misto comercial e pupa desidratada e moída de Bombyx mori em diferentes quantidades.

\begin{tabular}{lcccc}
\hline \multirow{2}{*}{ Quantidade de dieta } & \multicolumn{2}{c}{ Pupa desidratada } & \multicolumn{2}{c}{ Pólen } \\
\cline { 2 - 5 } & Macho & Fêmea & Macho & Fêmea \\
\hline 0,05 & $7 \mathrm{~b}^{*}$ & $9 \mathrm{~b}$ & $12 \mathrm{~b}$ & $10 \mathrm{~b}$ \\
0,1 & $9 \mathrm{a}$ & $12 \mathrm{ab}$ & $15 \mathrm{ab}$ & $14 \mathrm{ab}$ \\
0,2 & $12 \mathrm{a}$ & $15 \mathrm{ab}$ & $20 \mathrm{a}$ & $17 \mathrm{a}$ \\
0,5 & $15 \mathrm{a}$ & $15 \mathrm{ab}$ & $20 \mathrm{a}$ & $17 \mathrm{a}$ \\
1,0 & $25 \mathrm{a}$ & $21 \mathrm{a}$ & $25 \mathrm{a}$ & $25 \mathrm{a}$ \\
\hline
\end{tabular}

* Quantidade de insetos seguidos de mesma letra na coluna não diferem entre si pelo teste Meta - Análise para várias proporções em nível de 5\% de probabilidade de erro.
Apesar de a atração ter sido realizada a pequenas distâncias, evidencia-se que os insetos terão mais facilidade na localização do alimento. Além do mais, oferece condições que o inseto se desenvolva na dieta, aumentando suas populações. Portanto, os materiais testados se enquadram nas proposições de PARRA et al. (2002) para incremento do controle biológico no campo.

Como as tesourinhas apresentam um comportamento de grupo, estas dietas espalhadas em pontos estratégicos do campo poderiam aumentar a movimentação do predador, que iria se deslocar mais de uma planta a outra, aumentando assim a eficiência do combate a $\boldsymbol{S}$. frugiperda. Para que se concretize esta possibilidade, estudos adicionais deverão ser conduzidos com o propósito de avaliar, em campo, o efeito do fornecimento da dieta nas populações do predador e da praga. Assim, vários aspectos deverão ser avaliados, como proteção contra chuvas, alimentação por pássaros e outros insetos, etc.

Outra linha de investigação que merece atenção é a identificação de substâncias voláteis que eventualmente exerceram atração no predador. 


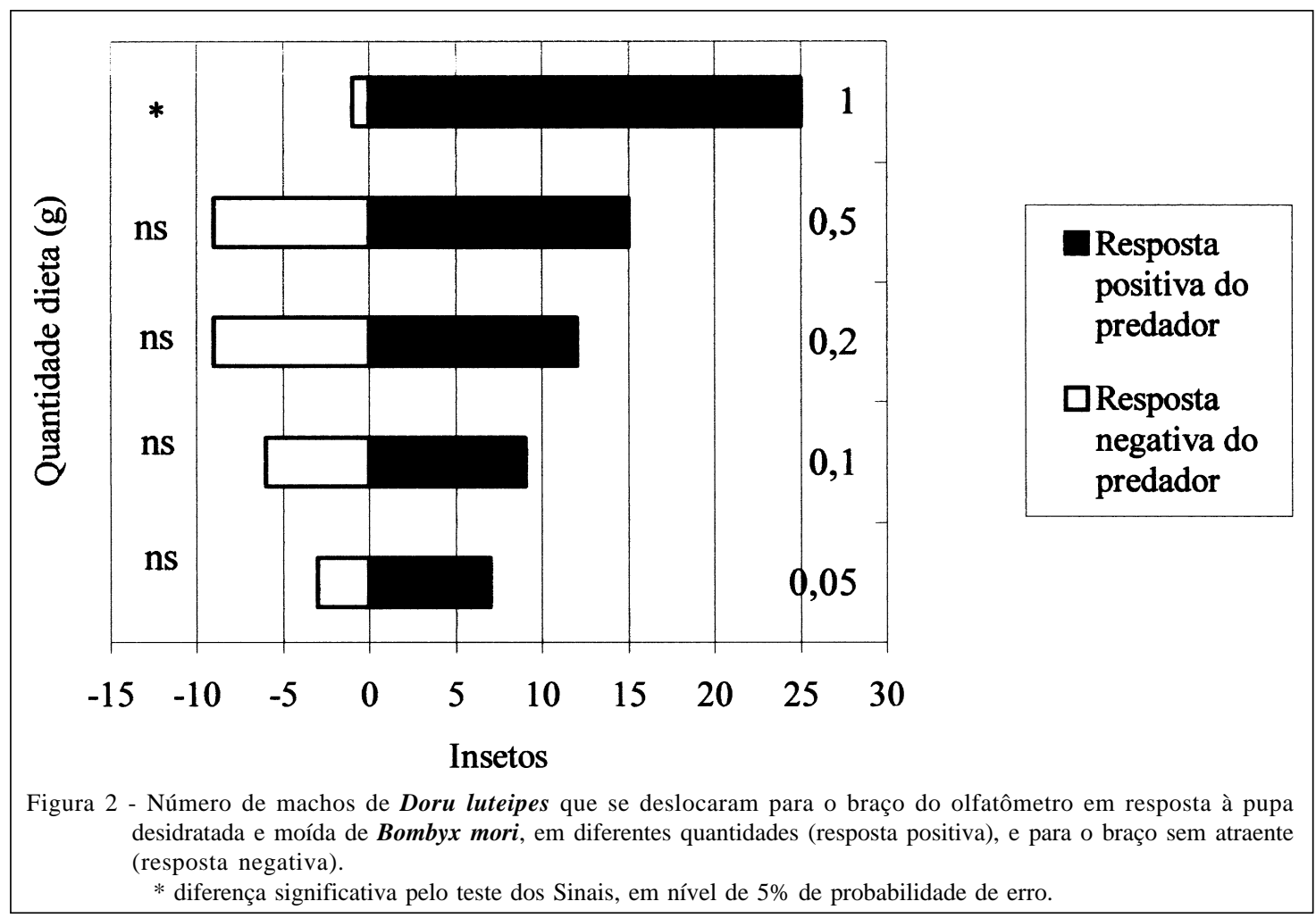

Avaliações anteriores caracterizaram também levedo de cerveja como atrativo para Doru sp. (GALBIATI et al., 1997), o que justifica avaliar o efeito combinado destas substâncias na atração do predador.

\section{CONCLUSÃO}

Pólen misto de plantas e farinha de pupa desidratada e moída de bicho-da-seda exercem atração

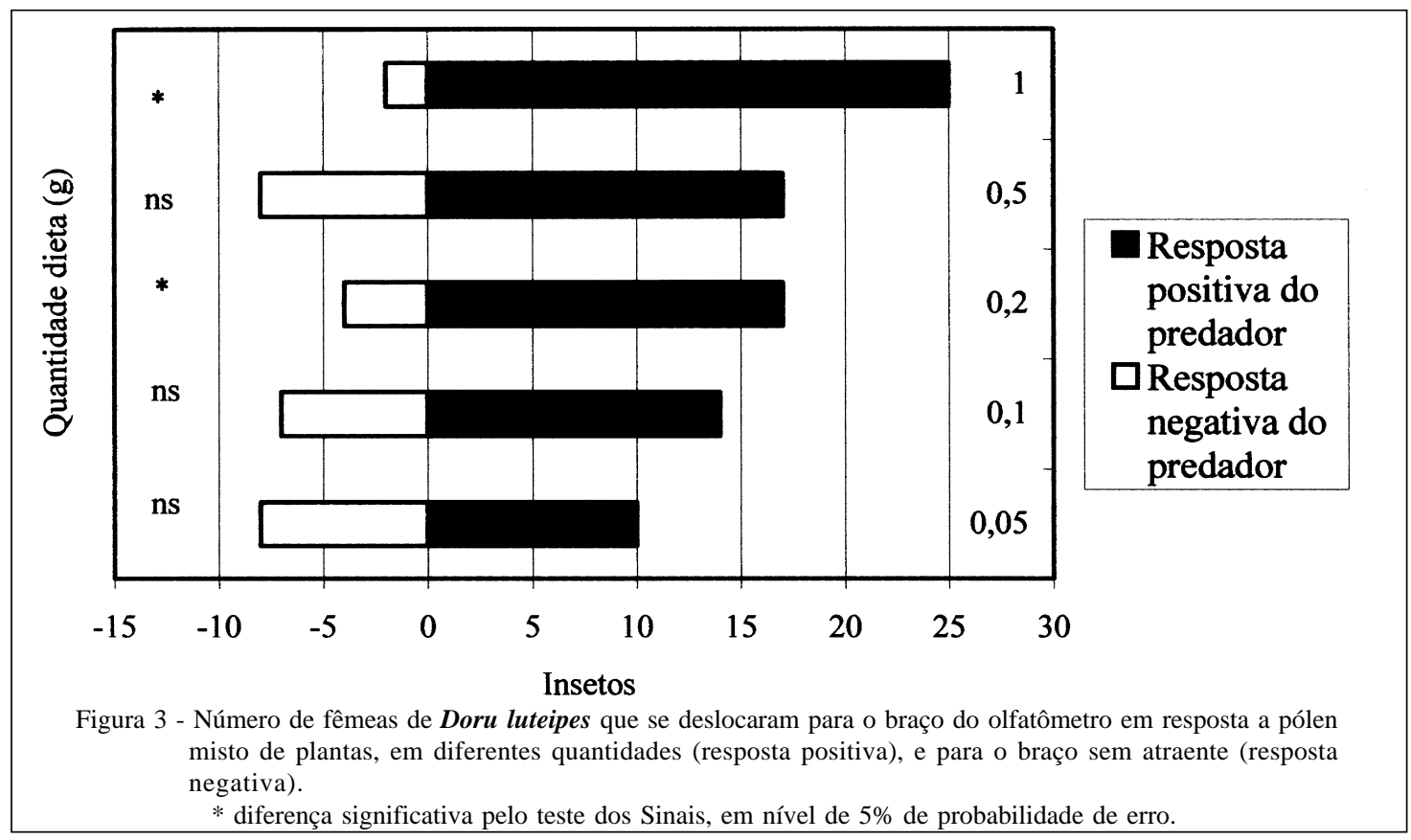

Ciência Rural, v.37, n.3, mai-jun, 2007. 


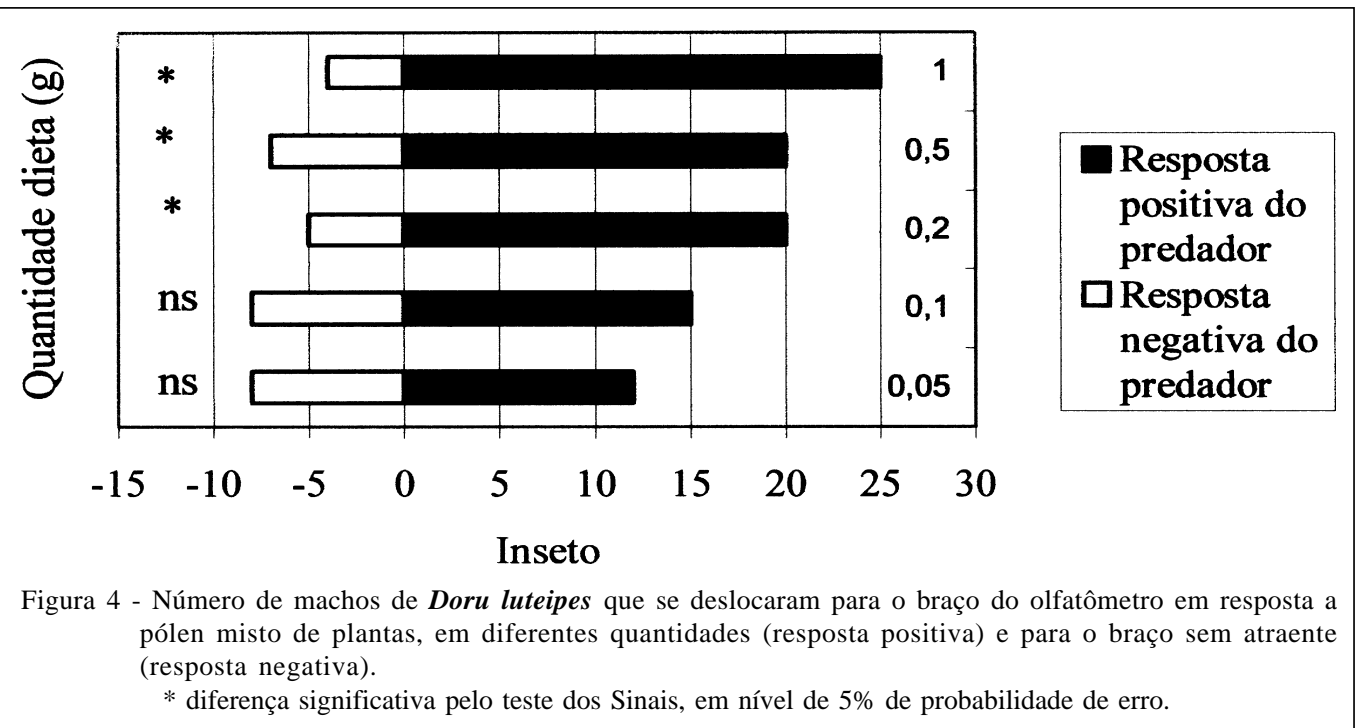

olfativa para o predador $\boldsymbol{D}$. luteipes, sendo, de maneira geral, essa atratividade aumentada com o aumento da quantidade dos referidos alimentos.

\section{AGRADECIMENTOS}

À professora Inês Fonseca, do Departamento de Agronomia da Universidade Estadual de Londrina, pela ajuda oferecida na análise estatística dos dados.

\section{REFERÊNCIAS}

ADLER, P.H. Sugar feeding of the adult corn earworm (Lepidoptera: Noctuidae) in the laboratory. Journal of Economic Entomology, v.82, p.1344-1349, 1989.

ALVARENGA, et al. Efeito do predador Doru luteipes (Scud.) sobre o crescimento populacional de Schizaphis graminum (Rond.) em diferentes genótipos de sorgo. Anais da Sociedade Entomológica do Brasil, v.25, p.137-140, 1996.

AYRES, M. et al. BioEstat 3.0.: aplicações estatísticas nas áreas de ciências biológicas e médicas. Belém: Sociedade Civil Mamirauá, 2003. p.291.

BARTLET, E. et al. The influence of glucosinolates and sugars on feeding by the cabbage stem flea beetle, Psylliodes chrysocephala. Entomologia Experimentalis et Applicata, v.73, p.77-83, 1994.

CAMPOS, A.R.; GRAVENA, S. Inseticidas, Bacillus thuringiensis e artrópodos predadores no controle da lagarta da maçã no algodoeiro. Anais da Sociedade Entomológica do Brasil, v.12, p.95-105, 1984.

CRUZ, I. Manejo integrado de pragas de milho com ênfase para o controle biológico. In: CICLO DE PALESTRAS SOBRE CONTROLE BIOLÓGICO DE PRAGAS, 4., 1995, Campinas, SP. Anais... Campinas: Sociedade Entomológica do Brasil, 1995, p. 48-92.
CRUZ, I. et al. Efeito do nível de saturação de alumínio em solo ácido sobre os danos de Spodoptera frugiperda (J. E. Smith) em milho. Anais da Sociedade Entomológica do Brasil, Londrina, v.25, p.293-297, 1996.

DITMAN, L.P.; CORY, E.N. The response of corn earworm moths to various sugar solutions. Journal of Economic Entomology, v.26, p.109-115, 1933.

GALBIATI, C. et al. Preferência de Doru sp. e Chrysopa externa (Hagen, 1861) por alimentação artificial em laboratório. Revista Ecossistema, v.22, p.24-26, 1997.

LEITE, L.G., LARA, F.M. Flutuação populacional de insetos e inimigos naturais associados à cultura da soja em Jaboticabal, SP. Anais da Sociedade Entomológica do Brasil, v.14 p.45-57, 1985.

PARRA, J.R.P. et al. Controle biológico no Brasil: parasitóides e predadores. São Paulo: Malone, 2002. 603p.

PASINI, A. Dieta artificial para criação de Doru luteipes (Scudder, 1876) (Dermaptera: Forficulidae) predador da lagarta do cartucho do milho, Spodoptera frugiperda (J.E.SMITH, 1797), em laboratório. 2002. 18f. Projeto de Pesquisa (Pós-Doutorado em Agronomia) - ESAUQ/USP, Piracicaba.

PASINI, A. et al. Técnica de criação de Doru luteipes (Scudder) e Doru lineare (Eschs) (Dermaptera: Forficulidae) em dietas natural e artificial. In: SIMPÓSIO DE CONTROLE BIOLÓGICO 8., 2003, Águas deSão Pedro. Anais... São Pedro: Simpósio de Controle Biológico, 2003a. p.153.

PASINI, A. et al. Dieta artificial para criação de Doru luteipes (Scudder) (Dermaptera: Forficulidae). In: SIMPÓSIO DE CONTROLE BIOLÓGICO, 8, 2003, Águas de São Pedro. Anais... São Pedro: Simpósio de Controle Biológico, 2003b. p.153.

WAQUIL, J.M. et al. Cultivo de milho: manejo integrado de pragas. Sete Lagoas: Embrapa, 2002. p.16. (Comunicado técnico, 50) 